Methods This study used data from the Survey of Health, Ageing and Retirement in Europe (SHARE) and English Longitudinal Study of Ageing (ELSA). We measured cognitive function by one key cognitive domain available in both surveys, namely episodic memory score (range 0-20). Sensory impairment was measured using self-reported hearing and vision quality in both SHARE and ELSA. Vision and hearing function in both surveys were each coded on a scale from 1 (excellent) to 5 (poor). We recoded the scores of hearing and vision function into two categories by combining the responses excellent, very good and good into 'good' sensory function and collapsing fair and poor vision into 'poor' sensory function. We defined sensory impairment as having poor sensory function and categorised it into three: no impairment, single impairment (vision or hearing), and dual impairment (vision and hearing).

Results Using growth curve analysis, we found that older adults with single and dual sensory impairment (hearing and/ or vision) were able to recall fewer words than those with no sensory impairment in final model in all 11 European countries included in this study, where the potential confounders were included. The cognitive trajectories of older adults with no sensory impairment followed curvilinear shapes, while those of older adults with single and dual sensory impairments showed more precipitous pattern trajectory of cognitive decline after the age of 50 in both surveys. Being female, having attained a higher level of education, having paid job and being relatively wealthy, were associated with higher cognitive function in older age both in SHARE and ELSA.

Conclusion These findings demonstrate that hearing and/or vision impairment is a marker for risk of cognitive decline that could inform preventative interventions to maximise cognitive health and longevity. Further studies are needed to investigate how sensory markers could inform strategies to improve cognitive ageing, including hearing and vision rehabilitative intervention in combination with healthy ageing interventions to promote social engagement, physical activity and positive health behaviours.

\section{P08 INCIDENCE OF DIAGNOSED DEMENTIA IN THE ENGLISH LONGITUDINAL STUDY OF AGEING IN ENGLAND: A 12- YEAR FOLLOW-UP}

${ }^{1} \mathrm{D}$ Cadar*, ${ }^{1} \mathrm{H}$ Davies, ${ }^{2} \mathrm{D}$ Llewellyn, ${ }^{3} \mathrm{GD}$ Batty, ${ }^{1} \mathrm{~A}$ Steptoe. ${ }^{1}$ Department of Behavioural Science and Health, University College London, London, UK; ${ }^{2}$ Mental Health Research Group, University of Exeter Medical School, University of Exeter, Exeter, UK; ${ }^{3}$ Department of Epidemiology and Public Health, University College London, London, UK

\subsection{6/jech-2017-SSMAbstracts. 110}

Background There has been a suggestion that the incidence of dementia in England has recently declined. Crucially, however, these estimates have not been based on nationally representative samples. Accordingly, we calculated dementia incidence, the temporal trends and, additionally, examined various socioeconomic and geographical predictors of its occurrence.

Methods Data from the English Longitudinal Study of Ageing (ELSA), a prospective cohort study at baseline known to be representative of the English population, were used to investigate the rates of dementia incidence over seven waves between 2002 and 2014.

Dementia was determined by doctor-diagnosis and the Informant Questionnaire on Cognitive Decline in the Elderly (score $>3.38$ indicative of dementia). Age- and sex-specific incidence rates of dementia were calculated for 5131 individuals aged 65 and older at study entry. Dementia incidence over time was examined by comparing age-specific incidence rates between two age-period-cohorts derived in ELSA using a median split in birth-year range (ELSA I: 1900-1925 and ELSA II: 1926-1950). The predictive value of education, wealth, geographical area, the index of multiple deprivations and the degree of urbanisation for dementia incidence were examined using Poisson regression.

Dementia was determined by doctor-diagnosis and the Informant Questionnaire on Cognitive Decline in the Elderly (score $>3.38$ indicative of dementia). Age- and sex-specific incidence rates of dementia were calculated for 5131 individuals aged 65 and older at study entry. Dementia incidence over time was examined by comparing age-specific incidence rates between two age-period-cohorts derived in ELSA using a median split in birth-year range (ELSA I: 1900-1925 and ELSA II: 1926-1950).

The predictive value of education, wealth, geographical area, the index of multiple deprivations and the degree of urbanisation for dementia incidence were examined using Poisson regression.

Results The average duration of follow-up was 7.7 years. As anticipated, there was a significant increase in the rates of dementia incidence with age, from 5.90 per 1000 person years at ages 65-69 to 31.59 at ages $85+$.

We observed an age-period-cohort effect on dementia incidence, with nearly $30 \%$ reduction in the incidence rates for participants of the same initial age group (75-79) at the study entry from ELSA I and II.

Higher rates of dementia incidence were observed for London $($ Rate $=15.52$, Incidence rate ratio $($ IRR $)=1.4395 \%$ confidence intervals (CI) 1.01-2.03) compared with Northern England, and for those in the lowest wealth quintiles (Rate $=14.61$, IRR $=2.18$, 95\% CI 1.55-3.06) compared with the highest.

Conclusion In a nationally representative sample, we observed a reduction in the age-specific dementia incidence over time. This incidence appeared to be patterned by group- and individual-level characteristics.

\section{P09 AFFECTIVE PROBLEMS AND MEMORY DECLINE: A SYSTEMATIC REVIEW AND META-ANALYSIS}

${ }^{1} \mathrm{~A}$ John*, ${ }^{1} \mathrm{U}$ Patel, ${ }^{2} \mathrm{M}$ Richards, ${ }^{1} \mathrm{R}$ Rusted, ${ }^{1} \mathrm{D}$ Gaysina. ${ }^{1}$ Psychology, University of Sussex, Brighton, UK; ${ }^{2}$ MRC Unit for Lifelong Health and Ageing, UCL, London, UK

\subsection{6/jech-2017-SSMAbstracts.111}

Background Previous evidence suggests that the presence of affective problems, such as depression and anxiety, may confer an increased risk for late-life dementia. However, the extent to which affective symptoms may influence memory decline in particular, even many years prior to the clinical threshold for a diagnosis of dementia, is not clear. The present study systematically reviews and synthesises the current evidence surrounding the association between depression and memory decline across the life course.

Methods An electronic search of PubMed, PsycInfo and ScienceDirect was conducted to identify studies on the association between depression and subsequent memory decline. Key inclusion criteria were prospective, longitudinal studies with a minimum follow-up period of one year. Cross-sectional, 
experimental, and clinical (case-control) studies were excluded. Reference lists of relevant papers were scanned for any additional articles of interest. Data extraction and methodological quality assessment using the STROBE checklist were conducted independently by two raters. Multi-level meta-analyses were conducted, with consideration of a number of potential moderators, including mean age of sample at baseline, length of follow-up, and quality of study.

Results After removal of duplicate references, all papers were screened for eligibility using a three-step process: 1) title screening $(n=20,954) ; 2)$ abstract screening $(n=981)$; and 3$)$ full text screening $(n=172)$. Inter-rater reliability at each stage of screening was $>90 \%$. A total of 17 studies (with 8 assessing depression as a binary variable and 9 assessing depression as a continuous variable) met eligibility criteria and had sufficient statistical information for extraction. The results of the meta-analyses will be presented and discussed with a focus on the effects of the key moderators that may influence the link between depression and memory decline, such as mean age at baseline, length of follow-up, and quality of study. Preliminary analyses suggest that affective problems significantly increase risk for subsequent decline in memory (Binary meta-analysis: $\mathrm{OR}=1.47 ; 95 \% \mathrm{CIs}=1.15,1.87, \mathrm{p}=0.002$; Continuous metaanalysis: $\quad \mathrm{B}=-0.007, \quad 95 \% \quad \mathrm{CIs}=-0.011,-0.002, \mathrm{p}=0.003)$. Conclusions Results of the present study improve current understanding of the temporal nature of the association between depression and memory across the life-course. This has important implications for the identification of individuals who are at a particularly high risk for accelerated decline in memory function and dementia.

\section{P10 DIET QUALITY, SARCOPENIA AND FRAILTY IN OLDER MEN: CROSS SECTIONAL ANALYSIS FROM THE BRITISH REGIONAL HEART STUDY}

${ }^{1} \mathrm{TJ}$ Parsons*, ${ }^{2} \mathrm{E}$ Papachristou, ${ }^{3}$ SE Ramsay, ${ }^{4} \mathrm{JL}$ Atkins, ${ }^{1} \mathrm{O}$ Papacosta, ${ }^{1} \mathrm{LT}$ Lennon, ${ }^{1} \mathrm{~S}$ Ash, ${ }^{5} \mathrm{PH}$ Whincup, ' $\mathrm{SG}$ Wannamethee. ${ }^{1}$ Primary Care and Population Health, UCL, London, UK; ${ }^{2}$ Institute of Education, UCL, London, UK; ${ }^{3}$ Institute of Health and Society, Newcastle University, Newcastle, UK; ${ }^{4}$ Epidemiology and Public Health, University of Exeter, Exeter, UK; ${ }^{5}$ Population Health Research Institute, St George's University of London, London, UK

\subsection{6/jech-2017-SSMAbstracts.112}

Background Frailty, a vulnerability to adverse health outcomes, and sarcopenia, a decline in muscle mass and strength or performance are associated with ageing. Frailty and sarcopenia predict increased mortality and hospitalisation, and sarcopenia often occurs with an increase in body fat known as sarcopenic obesity which elevates these risks further. Diet quality is well established as a predictor of mortality, but few studies have investigated diet quality in relation to frailty or sarcopenia, and findings are inconclusive. We have therefore examined the associations between diet quality, frailty and sarcopenic obesity categories.

Methods We used cross sectional data from community-dwelling men aged 71-91 years (British Regional Heart Study) in 2010-2012 recruited from 24 primary care practices. Men completed a food frequency questionnaire, from which the Healthy Dietary Index (HDI) and Elderly Dietary Index (EDI) were derived, and attended a physical examination. Frailty was based on the 5 components of the Fried frailty phenotype and we used a sarcopenic obesity classification which defines 4 groups; optimal, sarcopenic, obese or sarcopenic obese based on waist circumference and mid-arm muscle circumference.
We used logistic regression models to investigate whether diet quality was associated with frailty and sarcopenia and/or obesity.

Results $1331 / 3137$ men (42\%) had data for sarcopenia/obesity, all covariates and diet quality and 1119 men (36\%) for frailty, covariates and diet quality. After adjusting for age, social class, region of residence, smoking, alcohol consumption, cardiovascular disease and energy intake, men in the top quartile of the HDI score had a lower odds of being frail $(0.5895 \% \mathrm{CI}$ $0.34,0.96)$ compared with men in the bottom quartile, and men in the top quartile of either HDI or EDI had a lower odds of being obese compared with men in the bottom quartile $(0.5295 \%$ CI $0.33,0.84 \%$ and 0.57 95\% CI $0.38,0.86$ respectively). Neither the HDI or EDI was associated with sarcopenia or sarcopenic obesity, and the EDI was not associated with frailty.

Conclusion Higher diet quality based on both the HDI and EDI is associated with obesity but we found no evidence that diet quality is associated with sarcopenia in these elderly British men. However, our findings suggest that a higher diet quality as indicated by the HDI, a measure of adherence to WHO nutrient intake guidelines, might be relevant for the prevention or reversal of frailty.

\section{P11 THE ENDURING INFLUENCE OF CONTROLLING PARENTING ON PERSONAL MASTERY IN OLDER AGE}

FH Harkness*, M Stafford, T Cosco, M Richards. 'MRC Unit of Lifelong Health and Ageing, UCL, London, UK; ${ }^{2} M R C$ Unit of Lifelong Health and Ageing, UCL, London, UK; ${ }^{3} M R C$ Unit of Lifelong Health and Ageing, UCL, London, UK; ${ }^{4}$ MRC Unit of Lifelong Health and Ageing, UCL, London, UK

\subsection{6/jech-2017-SSMAbstracts.113}

Background Personal mastery is the subjective feeling of control over the events in one's own life. It is associated with healthy ageing, including better cardio-metabolic health, immune function and physical functioning. As an adult mastery is strongly associated with achievements of education, income and social class. However, within-group differences indicate that there could be other ways to feel in control. Mastery is theorised to be a self-concept first learnt in adolescence, and as such family may play a role in shaping it. Those whose parents support them psychologically and allow them appropriate freedom as an adolescent may grow up perceiving themselves to be in control, over and above tangible socio-economic resources.

Data The Medical Research Council National Survey of Health and Development (NSHD) is a representative sample of births in mainland Britain that occurred during a week in March 1946. Participants were $(n=1,037)$ study members who had provided data at ages $4,26,43$ and 68. Controlling parenting was measured using the Parental Bonding Instrument (PBI). This measures percieved parental levels of psychological control (e.g. invasiveness, overprotection).

The outcome was personal mastery assessed at age 68 using Pearlin's 7 item scale. An example item is, " I have little control over the things that happen to me." Multivariable regression analysis was used to test the association between psychologically controlling parenting and personal mastery age 68, controlling for childhood and adult socio-economic markers.

Results Higher perceived parental psychological control was associated with lower mean mastery -0.12 (95\% $0.20,-0.04$ ) 